\title{
Design and implementation of Smart Sensor Integrated Chair for Medical Diagnosis
}

\author{
Ms. M. Kiruthika, II ME (MPC), Mrs. K.Abhirami M.E., (Ph.D) \\ Assistant Professor, Department of CSE \\ Kings College of Engineering, Thanjavur, Tamil Nadu, India
}

\begin{abstract}
With the design of an Internet of Things (IoT) and telemedicine based health monitoring system- The Smart Chair. Sensors and associated hardware needed to monitor the vital physiological parameters of the human body are available on the chair, thereby leading to the idea of a Smart Chair. It enables the subject to be seated in a relaxed posture during the acquisition of physiological signals from various sensors attached to his/her body. The raw signals from the sensors are processed digitally by an onboard microcontroller and analyzed for any common abnormalities in the health parameters of the subject. The results are then transmitted to a personal computer. The data can be viewed at any later time by a doctor's computer that is connected to Internet. The Smart Chair also sends an SMS with all the health details to a remote doctor's phone in case of an emergency, thus facilitating telemedicine in rural areas. The key focus of the presented work is to propose the design of a chair that will be useful and easily affordable by the people of developing nations who have limited access to proper healthcare facilities. The results Presented show that the proposed system is definitely a low cost affordable solution for IoT based telemedicine system, as compared to existing systems
\end{abstract}

\section{Key Words: Internet of Things (IOT), Sensor.}

\section{INTRODUCTION}

Wireless sensor network and IoT has become an important technology in various industries with the healthcare sector being the most emerging of all. Remote monitoring of subjects is an innovative way to provide better, instantaneous and low cost access to healthcare. Good amount of research has been done in the field of telemedicine using mobile devices. With the availability of portable biomedical sensors and easy access to internet on mobile devices, medical devices on Internet of Things (IoT) are becoming increasingly common. Custom architectures for IoT based healthcare have also been proposed and the research is ongoing. However, most of the systems currently available in literature are designed to detect a particular physiological abnormality and are sometimes too expensive to be affordable by masses. Machines that monitor the common vital physiological parameters are under development using open source hardware and software. However, the ease of use for the end user is always a challenge. This includes the correct placement of the sensors and ease of operation of the biomedical device. The system architecture is available as open source, but the cost of complete kit is quite high because of the use of off the- shelf biomedical sensors/systems.

\section{LITERATURE SURVEY}

3.1 Model driven flexible design of a wireless body sensor network for health monitoring

The Wireless Body Sensor Network (WBSN) is a wireless network that is designed to allow communication among sensor nodes that are attached to a human body to monitor the body vital parameters and environment. The design and development of such WBSN systems for health monitoring have received a large amount of attention recently, in research studies and in industry. This attention is mainly motivated by costly health care and by recent advances in the development of miniature health monitoring devices as well as emerging technologies, such as the Internet of Things (IoT), which contribute to the main challenges of $5 \mathrm{G}$. 


\subsection{Routing Techniques in Wireless Sensor Networks: An Overview}

Due to the limited transmit power and other constraints; the transmission of data between two nodes in a Wireless Sensor Network (WSN) may have to be accomplished with the help of one or multiple relay nodes. Game theory has been proven very useful for accurately modelling the underlying strategic interactions between the sensor nodes and for providing the relays with incentives to forward the other nodes' data.

\subsection{Internet of things in industries: A survey}

Internet of Things (IoT) has provided a promising opportunity to build powerful industrial systems and applications by leveraging the growing ubiquity of radio-frequency identification (RFID), and wireless, mobile, and sensor devices. A wide range of industrial IoT applications have been developed and deployed in recent years. In an effort to understand the development of IoT in industries, this paper reviews the current research of IoT, key enabling technologies, major IoT applications in industries, and identifies research trends and challenges. A main contribution of this review paper is that it summarizes the current state-of-the-art IoT in industries systematically.

\subsection{IoT--from research and Innovation to Market Deployment}

The IoT will fuel technology innovation by creating the means for machines to communicate many different types of information with one another while contributing in the increased value of information created by the number of interconnections among things and the transformation of the processed information into knowledge shared into the Internet of Everything.

\subsection{Remote Wireless Patient Monitoring System}

One of the medical devices we found when we visit a hospital care unit such device is 'patient monitoring system'. This device (patient monitoring system) informs doctors and nurses about the patient's physiological signals. However, this device (patient monitoring system) does not have a remote monitoring capability, which is necessitates constant onsite attendance by support personnel (doctors and nurses). Thus, we have developed a Remote Wireless Patient Monitoring System using some biomedical sensors and Android OS, which is a portable patient monitoring. This device (Remote Wireless Patient Monitoring System) monitors the biomedical signals of patients in real time and sends them to remote stations (doctors and nurses android martphone and web) for display and with alerts when necessary.

\section{AIMS AND OBJECTIVES OF THE STUDY}

To monitor and gather the patient's physiological vital signs in a single sensor based component/ equipment. With the availability of portable biomedical sensors and easy access to internet on mobile devices, medical devices on Internet of Things (IoT) are becoming increasingly common one. Machines that monitor the common vital physiological parameters are under development using open source hardware and software. However, the ease of use for the end user is always a challenge. This includes the correct placement of the sensors and ease of operation of the biomedical device.

\section{PROPOSED METHODOLOGY}

In health care monitoring the patient's health conditions are need to be frequently watched. Nowadays the health care scheme is focus on the measurement and monitoring various biological parameters of patient's body like heart rate, oxygen saturation level in blood and temperature. Here the subject to be seated in a relaxed posture during the acquisition of physiological signals from various sensors attached to his/her body. The raw signals from the sensors are processed digitally by an onboard microcontroller and analyzed for any common abnormalities in the health parameters of the subject.

\section{Requirements}

PIC16F877A microcontroller POWER SUPPLIES

VALVE REGULATED LA-6V4.5 RECHARGEABLE BATTERY

BC546/547/548/549/550 SEMICONDUCTOR

$>$ RELAY

$>$ TEMPERATURE SENSOR LM35

$>$ HP03 SERIES OF CALIBRATED SENSOR MODULE

$>$ BUZZER ELECTROMAGNETIC

28/40/44-PIN ENHANCED MICROCONTROLLERS

FLASH

LM35 PRECISION

CENTIGRADE

TEMPERATURE SENSORS

HEART BEAT SENSOR 


\section{IMPLEMENTATION RESULTS}
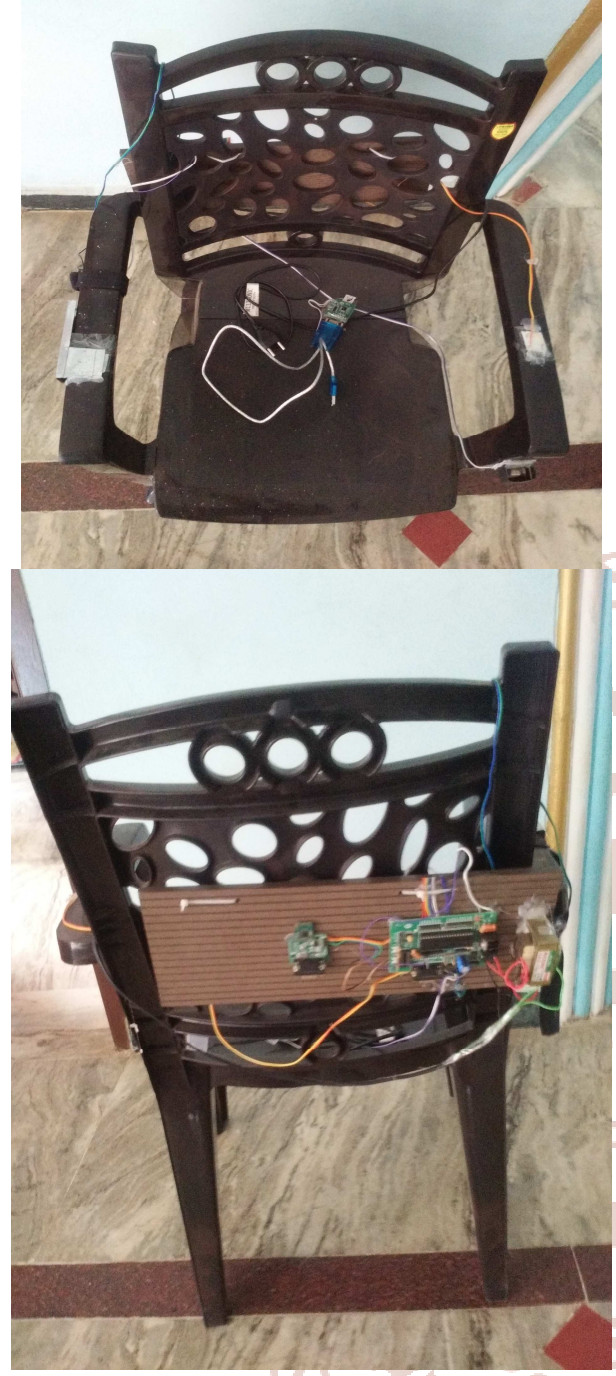

\section{CONCLUSION}

The design phase of the system, the cost was kept minimal. The key focus of the presented work is to propose the design of a product that will be useful and easily affordable by the people of developing nations who have limited access to proper healthcare facilities. The results presented show that the proposed system is definitely a low cost affordable solution for IoT based telemedicine system, as compared to existing systems.

\section{FUTURE ENHANCEMENT}

Future work involves attaching a keypad to the Smart Chair. A custom made web server will eliminate the constraint of creating an API key for each subject. This reduces the complexity of coding in the system. Having a keypad allows the user to enter his/her name and create a database in the web server for his/her health records.
9. REFERENCES

1. Da Xu, Li and $\mathrm{He}, \mathrm{Wu}$ and $\mathrm{Li}$, Shancang," Internet of things in industries: A survey," IEEE Transactions on Industrial Informatics, vol. 10, no. 4, pp. 2233-2243, 2014

2. Vermesan, O and Friess, P," IoT--From Research and Innovation to Market Deployment," River Publishers, 2014

3. Rotariu, Cristian and Pasarica, Alexandru and Costin, Hariton and Adochiei, Felix and Ciobotariu, Razvan," Telemedicine system for remote blood pressure and heart rate monitoring," in Proc. IEEE EHealth and Bioengineering Conference (EHB), pp. 1-4, 2011

4. Julio, Yair Enrique Rivera," Development of a Prototype Arduino- Mobile in Area of Telemedicine for Remote Monitoring Diabetic People (MAY 2015),' in Proc. IEEE Asia-Pacific Conference on Computer Aided System Engineering (APCASE), pp. 36-40, 2015

5. Vadillo, Silvia Blasco and Fernandez, Juan David Uceda and Perez, Maria del Mar Elena and Reboul, Jose Manuel Quero,” Design of a Remote Monitoring Platform for Telemedicine Systems Using New Generation Mobile Services," in Proc. IEEE Intl. conf. on Complex, Intelligent and Software Intensive Systems, pp. 643-648, 2008

6. UAHuntsville2013[Online]Available:http://portal. mhealth.uah.edu/public/index.ph

7. M. Chan, D. Estève, C. Escriba, E. Campo, A review of smart homes present state and future challenges, Computer Methods and Programs in Biomedicine pp. 55-81, 2008.

8. S. Sneha, U. Varshney, Enabling ubiquitous patient monitoring: model, decision protocols, opportunities and challenges, Decision Support Systems, vol.46, 606-619, 2009.

9. S. Koch, M. Hägglund, Health informatics and the delivery of care to older people, Maturitas,vol.63, no., pp. 195-199, 2009

10. M. Philipose, S. Consolvo, I. Smith, D. Fox, H. Kautz, D. Patterson, Fast, detailed inference of diverse daily human activities, in: Sixth International Conference on Ubiquitous Computing, 2004. 